\title{
Efecto de Tiempos de Retención Hidráulico de Agua, en un Sistema de Cultivo Experimental de un Paso para Trucha Arcoiris (Oncorhynchus mykiss)
}

Pablo A. Venegas, Catterina del P. Sobenes y Carlos M. Ortiz

Universidad Católica de la Santísima Concepción, Facultad de Ingeniería, Departamento de Ingeniería Ambiental y Recursos Naturales, Alonso de Ribera 2850, Concepción-Chile

(e-mail: pvenegas@ucsc.cl)

\begin{abstract}
Resumen
En un sistema experimental de cultivo de peces se probaron dos tiempos de retención hidráulico, superiores a lo normalmente utilizado, con el objeto de evaluar el efecto que se produce en el crecimiento de truchas arcoiris (Oncorhynchus mykiss) bajo condiciones normales de engorda (>700 g), en la calidad del agua de cultivo y en la productividad del sistema. Los resultados muestran que no hay diferencias en el crecimiento entre los grupos de truchas y que las variables de calidad de agua se mantienen en los rangos adecuados para su cultivo. En términos productivos, el uso de tiempos de retención de agua mayores en la engorda de truchas ( $3 \mathrm{~h}$ y $6 \mathrm{~h}$ ), permiten reducir su uso, manteniendo condiciones de calidad adecuadas, y aumentar la productividad al utilizar una carga de cultivo entre 2.5 a 5 veces más de lo que se utiliza normalmente.
\end{abstract}

\section{Water Hydraulic Retention Time Effects, in a single Pass Culture System for Rainbow Trout (Onchorhynchus mykiss)}

\begin{abstract}
On an experimental fish farming system, two hydraulic retention times, above the normally used values, were tested to evaluate the effect on the growth of rainbow trout (Oncorhynchus mykiss) under normal conditions of production $(>700 \mathrm{~g})$, on the quality of water and on the productivity of the system. The results show that there are no differences on the growth between the groups of trout and also that the variables of quality of water remain in ranks suitable for cultivation. In terms of productivity, the use of higher water retention times for the growing of the trout (3h and $6 \mathrm{~h}$ ) allows to reduce its use, keeping adequate quality conditions, and increasing productivity when using a cultivation load between 2.5 and 5 times more than normally used.
\end{abstract}

Keywords: aquaculture, trout farming, hydraulic retention time, water quality 


\section{INTRODUCCIÓN}

El cultivo de especies salmónidas en la fase de agua dulce, se realiza tradicionalmente en sistemas de cultivo de Un Paso, donde el agua ingresa y sale de las unidades de cultivo (reaceway o estanques circulares) sin ningún grado de re-uso (Colt, 1991; Colt et al., 1991; Lawson, 1995; Summerfelt et al., 2004). Esta forma de manejar el agua permite reducir la acumulación de residuos metabólicos (líquidos y sólidos) y el alimento no consumido, por medio de la dilución y arrastre del agua (Colt, 1991; Colt et al., 1991). Lo anterior exige el uso de importantes volúmenes de agua, los que pueden variar entre 375.000 a 657.000 litros para producir un kilo de pez por año (Pillay, 1982; Colt, 1991; Piedrahita, 2003). No obstante, la disponibilidad de agua con una calidad adecuada para acuicultura ha ido disminuyendo paulatinamente, debido al aumento de la demanda de agua orientada para otros usos productivos (turismo, agricultura, ganadería, forestación, crecimiento de las ciudades, etc.), el crecimiento de la industria acuícola y la contaminación desde distintas fuentes de agua. Esto ha obligado a la industria acuícola al desarrollo de tecnologías y sistemas para el manejo del agua que permitan disminuir su consumo, manteniendo o aumentando el rendimiento productivo de los cultivos (Piedrahita, 2003). Este manejo ha considerado aumentar el tiempo de retención hidráulico del agua en las unidades de cultivo y por consiguiente la mejora en los sistemas para su filtración y acondicionamiento, permitiendo la aparición de sistemas de cultivo con diferentes grados de re-uso del agua (en serie, parcial y recirculación), los que por medio de algún nivel de tratamiento logran aumentar su "vida útil". Según Piedrahita (2003) y Summerfelt et al. (2004) , el uso de este tipo de sistema reduce el consumo de agua por kilo de pez por año a 88.000 I para un sistema de re-uso en serie, donde el agua pasa de una a otra unidad de cultivo antes de ser eliminada; a 10.500 I para uno de re-uso parcial, donde el agua es recirculada continuamente y su calidad se mantiene sólo por oxigenación y dilución y; a 3.300 I en sistemas de recirculación, donde la calidad del agua se mantiene por tratamientos físicos, químicos, biológicos y por el ingreso de pequeños volúmenes de agua fresca.

Las necesidades de agua en los sistemas de cultivo de un paso, dependen de la relación entre los requerimientos de calidad de agua de la especie y de la tasa con que ésta se deteriora durante su paso por las unidades de cultivo. De esta forma, la calidad del agua y por lo tanto su cantidad se controla con el tiempo de retención hidráulico (TRH) en el sistema de cultivo. Para Colt (1991), el TRH para estos sistemas y especialmente en peces de agua fría, varía entre $0,17 \mathrm{~h}-0,34 \mathrm{~h}$ si el agua no es aireada y entre $0,48 \mathrm{~h}-0,96 \mathrm{~h}$ si se utiliza oxígeno puro. Sin embargo, en base a las estimaciones realizadas por Piedraita (2003), sobre la carga contaminante del agua efluente de los sistemas de un paso, las concentraciones de los compuestos de desecho (nitrógeno, fósforo y sólidos suspendidos totales), se encuentran bajo los límites adecuados para el cultivo de truchas, lo que permitiría aumentar el TRH para estos sistemas de cultivo sin afectar el normal desarrollo de la especie. Así, sería factible aumentar los volúmenes de producción de un centro de cultivo para un mismo volumen de agua disponible, aumentando su productividad y facilitando la utilización de fuentes de agua que por su bajo caudal disponible (pozos o riachuelos), no permiten actualmente el desarrollo de una actividad acuícola económicamente rentable.

En base a lo anterior, la presente investigación evaluó el efecto que dos tiempos de retención hidráulico de agua (TRH), aplicados a un sistema de cultivo experimental de un paso, genera sobre el crecimiento de un grupo de trucha arcoíris bajo condiciones normales en su etapa de engorda $(O$. mykiss). Además, se evaluó el efecto en la productividad del sistema, medido como carga de cultivo, y en las variables de calidad de agua: total de amonio nitrogenado (TAN), nitrito nitrogenado $\left(\mathrm{NO}_{2}-\mathrm{N}\right)$, nitrato nitrogenado $\left(\mathrm{NO}_{3}-\mathrm{N}\right)$ y sólidos suspendidos totales (SST).

\section{MATERIALES Y MÉTODOS}

Las experiencias de tiempos de retención hidráulica $(T R H)$ se realizaron en las instalaciones del Laboratorio de Ingeniería Acuícola de la Universidad Católica de la Santísima Concepción, durante los meses de enero y febrero del año 2007. Para las experiencias se instaló un sistema de cultivo en la modalidad de un paso, con un mecanismo de ingreso y descarga de agua que permitiera operar simultáneamente con dos TRH. Durante 60 días, dos grupos de trucha arcoíris fueron cultivadas con 
distintos TRH, registrando periódicamente su crecimiento y mortalidad y los cambios en las variables de calidad del agua.

El Sistema de un paso (ver figura 1), estaba compuesto por dos estanques de $1 \mathrm{~m}^{3}$ cada uno, que contenía el agua fresca ubicadas sobre una plataforma de madera de 0,5 $\mathrm{m}$ de altura. El agua era distribuida desde los estanques de acopio de agua fresca hacia un estanque ecualizador de $35 \mathrm{I}$, desde donde era redistribuida a las unidades de cultivo por cuatro bombas sumergibles de 6 watts de potencia cada una. Las unidades de cultivo estaban compuestas por cuatro estanques rectangulares de $0,35 \mathrm{~m}^{3}$ de capacidad ( $1 \mathrm{~m}$ de largo por $0,7 \mathrm{~m}$ de ancho y 0,5 $\mathrm{m}$ de profundidad), dos para cada TRH, fabricados en plástico laminado y reforzado con fibra de vidrio (PRFV) y con un desagüe inferior de PVC de $32 \mathrm{~mm}$ de diámetro (ver Fig. 1). Los estanques presentaban una altura de agua $0,24 \mathrm{~m}$, generando un volumen útil de $0,17 \mathrm{~m}^{3}$ en cada uno. El nivel del agua fue controlado con un tubo de PVC de $32 \mathrm{~mm}$ conectado al desagüe del estanque que contenían a los peces con piedras difusoras de cuarzo de poro medio para la aireación.

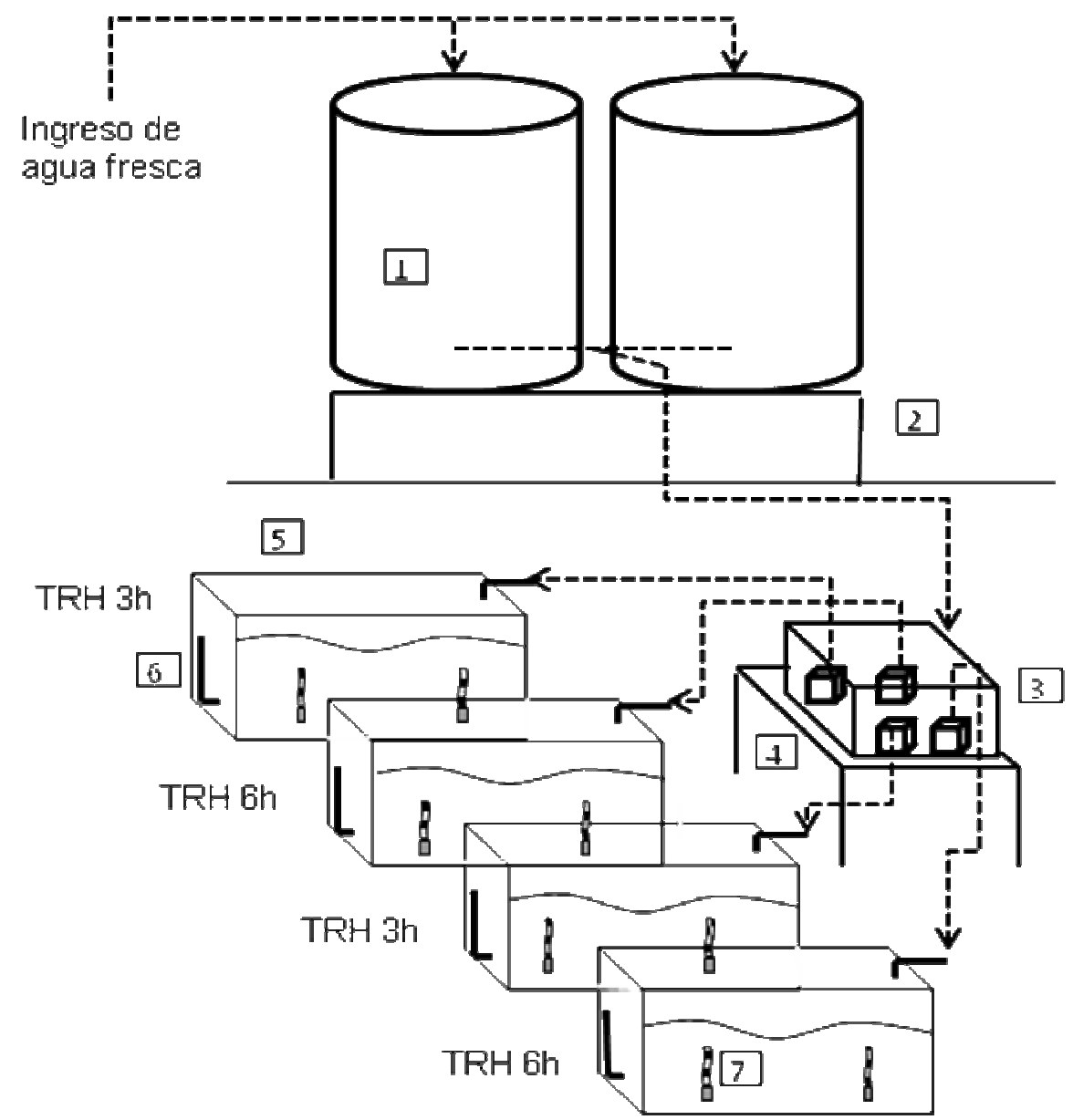

Fig.1: Sistema de Cultivo experimental de un paso y recambio de agua. (1) Estanques de acopio agua fresca, (2) plataforma, (3) estanque ecualizador, (4) bombas sumergibles, (5) unidades de cultivo, (6) tubo PVC desagüe y (7) difusores de poros para aireación.

La experiencia de cultivo se realizó con trucha arcoíris en su fase de engorda, peso medio de $723 \pm$ $133 \mathrm{~g}$, provenientes de una piscicultura de Chile central y que se mantienen en el Laboratorio de la Universidad, las cuales, previo al inicio de las pruebas, fueron aclimatadas durante una semana en las unidades de cultivo. Durante la experiencia se utilizó una densidad de cultivo normal para el tamaño de los especie y sistema de producción [15 a $40 \mathrm{~kg} / \mathrm{m}^{3}$ (Colt et al., 1991)], y un nivel de alimentación diaria equivalente a $1 \%$ del peso de la biomasa en los estanques.

La elección de los TRH sometidos a prueba se basó en la búsqueda de opciones de reducción del consumo de agua y en la necesidad de evitar que el deterioro de su calidad durante la experiencia, 
producto de su tiempo de retención en los estanques de cultivo, generara condiciones adversas o tóxicas para la especie, es decir, se sobrepasaran los rangos de variación definidos como normales. Así, se considero como limitante para definir un TRH al total de amonio nitrogenado disuelto en el agua, que para algunos autores (Bernabé, 1991; Lawson, 1995; Timmons et al., 2002; Lyssenko y Wheaton, 2006), no debería exceder la concentración de $1 \mathrm{mg} / \mathrm{l}$ en trucha arcoíris. De esta forma, se realizó un balance de masa para estimar el máximo TRH que no sobrepase la concentración de TAN sugerida y otro tiempo de retención superior que permita apreciar su posible efecto negativo. Las estimaciones indicaron que para una densidad de cultivo de $30 \mathrm{~kg} / \mathrm{m}^{3}$, una cantidad de alimento diaria a entregar equivalente al $1 \%$ de la biomasa de cada grupo, un volumen de $0,17 \mathrm{~m}^{3}$ de cada estanque y una producción diaria de TAN equivalente al 3\% del alimento entregado (Lawson, 1995), un TRH de 3h generaría una concentración máxima de amonio nitrogenado en el agua igual de 1,11 $\mathrm{mg} / \mathrm{l}$, similar a lo recomendado, y 2,22 mg/l para un TRH de $6 \mathrm{~h}$ que fue utilizado como contraste.

Para el recambio de agua fresca se utilizaron dos estanques acumuladores de agua fresca de $1 \mathrm{~m}^{3}$, montados sobre una plataforma de madera de $0,5 \mathrm{~m}$ de altura (ver Fig. 1). El agua llegaba al estanque de ecualización por gravedad desde los estanques de acumulación, en donde 4 bombas sumergibles alimentaban a cada estanque de cultivo, dos con un caudal de ingreso de agua de 0,94 $\mathrm{l} / \mathrm{min}$ y una velocidad en las tuberías de $0,02 \mathrm{~m} / \mathrm{s}$ (TRH de $3 \mathrm{~h}$ ) y los otros con un caudal de $0,47 \mathrm{l} / \mathrm{min}$ y una velocidad de $0,01 \mathrm{~m} / \mathrm{s}$ (THR de $6 \mathrm{~h}$ ). La descarga de agua de cada estanque de cultivo fue controlada con los tubos de desagüe, asegurando un TRH constante, el que se calculó de la siguiente manera:

$T R H=\frac{V}{Q}$

Donde $V$ es el volumen del sistema de cultivo (I) y $Q$ el caudal de agua que ingresa $(I / h)$.

\section{Operación y mediciones en el sistema de un paso}

Los peces de cada experiencia fueron alimentados diariamente con una cantidad de alimento definida y equivalente al $1 \%$ de la biomasa de cada estanque, la que se entregaba fraccionada en un $60 \%$ en la mañana y el resto en la tarde. No se considera la alimentación a saciedad, entregando alimento en función del comportamiento de los peces, pero con un máximo de un $1 \%$ diario. El alimento fue estimado en función a la biomasa de cada estanque, la que era actualizada diariamente por medio del coeficiente de crecimiento por unidad térmica (TGC), obtenidos con los muestreos biológicos propios. Las características del alimento suministrado fue a base de harina y aceite de pescado con $42 \%$ de proteínas, calibre $9 \mathrm{~mm}$ y con $80 \mathrm{ppm}$ de astaxantina, marca Ewos. Los individuos fueron pesados y medidos cada 15 días, calculando en cada caso la tasa de conversión de alimento (F.C.), el factor de condición (K), porcentaje de crecimiento en el período (PCP) y el coeficiente de crecimiento por unidad térmica (TGC) (Cho, 1992), basado en las ecuaciones siguientes:

$\% P . C .=\frac{W}{(A e)}$

Donde: \%P.C. es el porcentaje peso cuerpo, W es el peso de los peces en gramos, Ae es el alimento entregado por día en gramos.

$T G C=\frac{\left(W_{f}^{1 / 3}-W_{i}^{1 / 3}\right)}{\sum T^{*} d} * 1000$

Donde: TGC es el coeficiente de crecimiento por unidad térmica, $W_{f}$ es el peso final del pez en gramos, $W_{i}$ es el peso inicial del pez en gramos, $T$ es la temperatura media diaria en grados Celsius $\left({ }^{\circ} \mathrm{C}\right)$ y d es el número de días entre el día de medición del peso inicial $W_{i}$ y el peso final del pez $W_{f}$. 
P.C.P. $=\frac{W f-W i}{W i} * 100$

Donde: P.C.P es el porcentaje de crecimiento del período, $W_{f}$ es el peso del pez al final del período en gramos y $W_{i}$ es el peso del pez al inicio del período en gramos.

F.C. $=\frac{\text { Aed }}{\left(W_{f}-W_{i}\right)}$

Donde F.C. es la tasa de conversión de alimento, Aed es el alimento entregado al pez durante d días en gramos, $W_{f}$ es el peso final del pez en gramos, $W_{i}$ es el peso inicial del pez en gramos.

$K=\frac{W}{L^{3}} * 100$

Donde $\mathrm{K}$ es el factor de condición, $\mathrm{W}$ es el peso del pez en gramos y $\mathrm{L}$ es la longitud del pez en $\mathrm{cm}$.

Con el objeto de medir la productividad del sistema de cultivo, en términos de su eficiencia en el uso del agua, se determinó al final de la experiencia la Carga de Cultivo (C) para cada TRH, según la ecuación reportada por Lawson (1995).

$C=\frac{D^{*} 0,06}{R}$

Donde $C$ es la carga de cultivo en $\mathrm{kg}$ de pez/l/min; $D$ es la densidad de cultivo $\left(\mathrm{kg} / \mathrm{m}^{3}\right)$; $\mathrm{R}$ es el número de cambios de agua por hora en las unidades de cultivo; y 0,06 es el factor de conversión para las unidades de tiempo en sistema métrico.

Durante los 60 días de la experiencia se midieron y registraron las siguientes variables de calidad del agua de cultivo (Tabla 1).

Tabla 1: Variables de calidad de agua registradas, frecuencia de medición y equipos o procedimientos utilizados.

\begin{tabular}{|c|c|c|}
\hline Variable & Equipo o Procedimiento & $\begin{array}{l}\text { Frecuencia de } \\
\text { medición }\end{array}$ \\
\hline Acidez $(\mathrm{pH})$ & pHmetro Hanna, Modelo HI 98128 & \multirow{6}{*}{$\begin{array}{l}2 \text { veces al día (9:00 } \\
\text { a.m. y 6:00 p.m.) en } \\
\text { todos los estanques }\end{array}$} \\
\hline Oxígeno Disuelto $\left(\mathrm{O}_{2} \mathrm{mg} / \mathrm{l}\right)$ & \multirow{2}{*}{ Oxigenómetro oxi 330 iset, Marca WTW } & \\
\hline Temperatura $\left({ }^{\circ} \mathrm{C}\right)$ & & \\
\hline $\begin{array}{l}\text { Total Amonio Nitrogenado } \\
\text { (TAN mg/l) }\end{array}$ & \multirow{3}{*}{ Fotómetro Hanna Modelo C200 } & \\
\hline Amonio $\left(\mathrm{NH}_{3}-\mathrm{N} \mathrm{mg} / \mathrm{l}\right)$ & & \\
\hline Nitrito $\left(\mathrm{NO}_{2}-\mathrm{N} \mathrm{mg/l}\right)$ & & \\
\hline $\begin{array}{ll}\text { Sólidos } & \text { Suspendidos } \\
\text { Sedimentales }(\mathrm{mg} / \mathrm{l})\end{array}$ & Cono Imhoff & 1 vez al día (9:00 \\
\hline $\begin{array}{l}\text { Sólidos Suspendidos no } \\
\text { Sedimentales }(\mathrm{mg} / \mathrm{l})\end{array}$ & APHA (1998) & $\begin{array}{l}\text { a.m.) en un estanque } \\
\text { por experiencia }\end{array}$ \\
\hline Sólidos Disueltos (mg/l) & & \\
\hline
\end{tabular}

Para evaluar si los distintos THR generaron una diferencia significativa sobre el crecimiento de los grupos de truchas, los datos de muestreo de peso fueron sometidos a una regresión lineal para obtener los parámetros de las rectas. Posteriormente se realizó una prueba $\mathrm{F}$ para comparar las varianzas y finalmente un prueba t para determinar la posible igualdad entre las pendientes. Además, 
para cada muestreo quincenal, se realizó una prueba $\mathrm{t}$ con el objeto de determinar si existía diferencia significativa entre el peso medio con un TRH de $6 \mathrm{~h}$ y $3 \mathrm{~h}$.

Para evaluar el efecto sobre las variables de calidad de agua, se realizó con los datos obtenidos un análisis de varianza dentro y entre los tratamiento de TRH $6 \mathrm{~h}$ y $3 \mathrm{~h}$. Además, para los análisis que dieron diferencia significativa se aplicó una prueba t para determinar se existía diferencia entre las concentraciones de cada variable de calidad de agua, agrupadas por semana, entre los tratamiento de TRH de $6 \mathrm{~h}$ y $3 \mathrm{~h}$.

Previo a la aplicación de los test estadísticos indicados, se realizó un test Shapiro-Wilk para conocer la normalidad de los datos, con un nivel de significancia de $\alpha<0,05$. Para las pruebas estadísticas se utilizó el Software XLSTAT 5.1 en su versión de Demostración Libre

Finalmente, para evaluar el efecto de los $\mathrm{TRH}$, se realizó una comparación de la productividad de distintos sistemas de cultivo de un paso según lo reportado por distintas investigaciones.

\section{RESULTADOS Y DISCUSIÓN}

\section{Efecto del TRH sobre el crecimiento}

La ecuación que explica el Peso Medio (PM) v/s tiempo para un TRH de 6 horas es $P M_{T R H 6}=736,9+5,59 * t$ con $\mathrm{R} 2=0,99$ y para 3 horas es $P M_{T R H 3}=736,6+4,15 * t$ R2 $=0,97$. Las varianzas son estadísticamente iguales $(\mathrm{F}=1,78$ a un $5 \%$ ) y se probó el paralelismo entre ambas pendientes de manera de comprobar si ambas tasas de crecimiento eran iguales para ambos $\mathrm{TRH}$. En esta prueba se acepta la hipótesis nula de igualdad de pendientes a un $1 \%$ de error siendo $t=1,93$. Así, el análisis de los resultados muestra que no existe diferencia significativa en el crecimiento de los individuos, si éstos se encuentran en un sistema de un paso con un TRH agua de 3 horas ó 6 horas y una densidad de cultivo superior a los $40 \mathrm{~kg} / \mathrm{m}^{3}$, sobre la base de las condiciones de cultivo detalladas en la Tabla 2.

Tabla 2: Condiciones experimentales, factor de conversión (FC), factor de condición (K) y crecimiento (TGC) obtenidos en el periodo de estudio.

\begin{tabular}{|c|c|c|c|c|}
\hline Condiciones experimentales & $\begin{array}{c}\text { Estanque } 1 \\
\text { THR } 6 \mathrm{~h}\end{array}$ & $\begin{array}{c}\text { Estanque } 4 \\
\text { THR } 6 \mathrm{~h}\end{array}$ & $\begin{array}{c}\text { Estanque } 2 \\
\text { THR } 3 \mathrm{~h}\end{array}$ & $\begin{array}{c}\text { Estanque } 3 \\
\text { THR 3h }\end{array}$ \\
\hline Número de peces & 7 & 7 & 7 & 7 \\
\hline$\%$ Mortalidad & 0 & 0 & 0 & 0 \\
\hline Peso medio inicial $(\mathrm{g})$ & $704 \pm 99$ & $753 \pm 205$ & $709 \pm 135$ & $729 \pm 122$ \\
\hline Peso medio final $(\mathrm{g})$ & $1049 \pm 124$ & $1093 \pm 253$ & $949 \pm 260$ & $1013 \pm 199$ \\
\hline Factor de Condición inicial (K) & 1,268 & 1,373 & 1,195 & 1,163 \\
\hline Factor de Condición final (K) & 1,490 & 1,620 & 1,459 & 1,525 \\
\hline Densidad inicial $\left(\mathrm{kg} / \mathrm{m}^{3}\right)$ & 29,0 & 31,0 & 29,2 & 30,0 \\
\hline Densidad final $\left(\mathrm{kg} / \mathrm{m}^{3}\right)$ & 43,2 & 37,4 & 39,1 & 41,7 \\
\hline Biomasa ganada $(\mathrm{g})$ & 2416 & 2377 & 1679 & 1992 \\
\hline Alimento consumido (g) & 3454 & 3157 & 2876 & 3168 \\
\hline Factor de Conversión (FC) & 1,43 & 1,33 & 1,71 & 1,59 \\
\hline Temperatura Promedio $\left({ }^{\circ} \mathrm{C}\right)$ & $18,2 \pm 1,1$ & $18,3 \pm 1,1$ & $18,3 \pm 1,2$ & $18,3 \pm 1,2$ \\
\hline Número de días experiencia & 60 & 60 & 60 & 60 \\
\hline TGC & 1,158 & 1,096 & 0,826 & 0,950 \\
\hline$\%$ crecimiento del período & 49 & 45 & 34 & 39 \\
\hline
\end{tabular}

Los coeficientes de crecimiento térmico (TGC) obtenidos en esta experiencia variaron entre 0,000830,00116 , lo que indica un crecimiento en peso entre un $49 \%$ y $34 \%$ en los individuos (Tabla 2 ). Los TGC obtenidos puede ser considerados bajos si se compara con los valores reportados para trucha arcoíris (O.mykiss) por Cho (1992), entre 1,53-1,74 y Summerfelt et al. (2004), entre 1,32-2,18. Esta 
diferencia puede ser atribuida a la temperatura promedio del agua durante la experiencia, $18,3^{\circ} \mathrm{C}$ $\pm 1,2^{\circ} \mathrm{C}$, superior a lo recomendado para la especie $10^{\circ} \mathrm{C}-16^{\circ} \mathrm{C}$ (Piper et al., 1982), y adicionalmente a la diferencia en talla de los ejemplares utilizados en la experiencia. Sin embargo, Jobling et al. (1994), indican que el principal inconveniente en el uso del TGC para la comparación o proyección del crecimiento de un lote de peces, es su variabilidad en función de la temperatura del agua, así el valor característico de TGC es relativamente estable dentro del rango de temperatura normal para el cultivo de una especie y tiende a disminuir hacia su extremo superior, situación que se presentaría en este caso. Además, los factores de condición $(\mathrm{K})$ presentaron un comportamiento creciente durante toda la experiencia (Tabla 2), $18 \%$ para $6 \mathrm{~h}$ y $26 \%$ para $3 \mathrm{H}$, lo que indicó un aumento de peso proporcionalmente mayor a la longitud de los individuos, situación que para Jobling (2003) generaría una subestimación del valor final del TGC. La figura 2, muestra la evolución del crecimiento de los individuos durante la experiencia. Es posible observar una tendencia positiva y con tasas de crecimiento similares entre los dos tratamientos utilizados (error estándar), aunque se observa una leve disminución de la tendencia para un tiempo de retención hidráulico de 3 horas (TRH 3h).

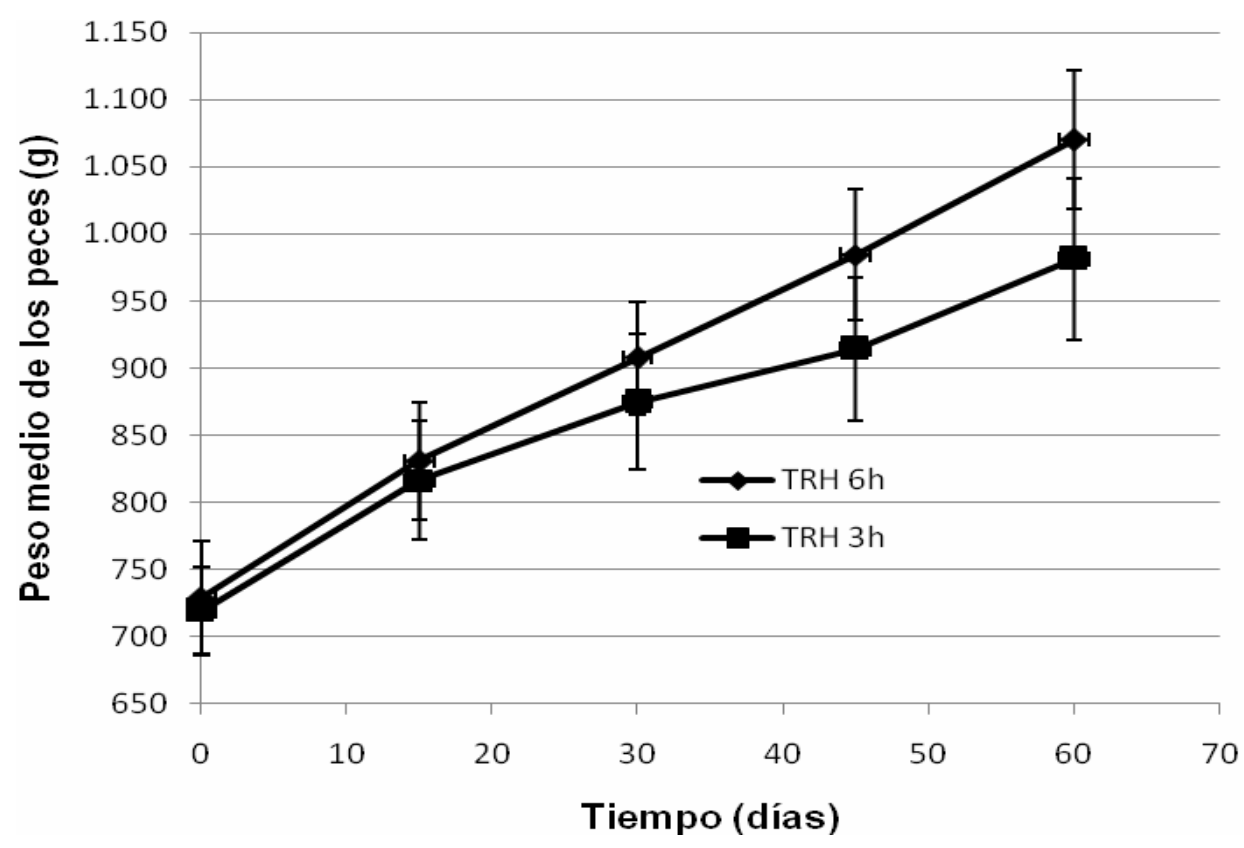

Fig. 2: Evolución del crecimiento en peso de las truchas durante la aplicación de distintos tiempos de retención hidráulica $(\mathrm{TRH})$ de agua. Se indica el error estándar $(n=14)$

En relación al comportamiento del consumo de alimento, este fue disminuyendo paulatinamente durante la experiencia, obligando a alimentar solamente hasta que los peces reaccionaban a la caída del pellet en el agua, desde un máximo definido de un $1 \%$ PC hasta $0,53 \%$ PC, valor obtenido más bajo. Esto que puede ser atribuido a la temperatura del agua que habría afectado la eficiencia de alimentación, asimilación y crecimiento de los individuos, lo que concuerda con lo descrito por Tucker y Robinson (1990) y Meade (1991), quienes atribuyen estos problemas al cultivo de una especie en rangos de temperatura mayores a lo recomendado.

\section{Efecto del tiempo de retención hidráulica (TRH) en la calidad de agua}

Los resultados indican que no existió diferencia significativa dentro de cada tratamiento (experiencias y su réplica) para la variación y valor medio de las concentraciones de total amonio nitrogenado (TAN), amonio $\left(\mathrm{NH}_{3}-\mathrm{N}\right)$, nitrito $\left(\mathrm{NO}_{2}-\mathrm{N}\right)$, oxígeno disuelto $\left(\mathrm{O}_{2}\right)$, sólidos suspendidos totales $(\mathrm{SST})$ y disuelto, y para los valores de $\mathrm{pH}$ y temperatura. Los valores máximos, medios y mínimos se detallan en la Tabla 3.

De igual forma, no se observó diferencia significativa entre los tratamientos para la variación y valor medio de las concentraciones de $\mathrm{NH}_{3}-\mathrm{N}$, TSS, sólidos disueltos y totales, y entre $\mathrm{pH}$ y temperatura del agua. Sin embargo, sí se observó diferencia significativa entre los tratamientos en los niveles de 
TAN ( $F=138,3$ a un $5 \%$ ) y $\mathrm{NO}_{2}-\mathrm{N}(\mathrm{F}=17,14$ a un $5 \%)$ (ver figuras 3 y 4$)$, siendo la experiencia con un $\mathrm{TRH}$ de $6 \mathrm{~h}$ la que presentó valores medios más altos (Tabla 3), lo que sería explicado por la menor dilución de contaminantes que presenta este tratamiento. La variable $\mathrm{NO}_{2}-\mathrm{N}$ presentó desde la cuarta semana de la experiencia, en ambos TRH, un aumento de su concentración en el agua de cultivo (Fig. 4), acompañado de una disminución de la concentración de TAN (fig. 3). Esto podría indicar la presencia de una colonia de bacterias nitrificantes que se habría desarrollo y asentado en las tuberías y estanques de cultivo durante la experiencia, oxidando el amonio disponible a nitrito, concordando con lo propuesto por Summerfelt et al. (2004) para explicar una situación similar en un sistemas de re-uso parcial. La mayor concentración de $\mathrm{NO}_{2}-\mathrm{N}$ en la experiencia con un TRH de $6 \mathrm{~h}$, se explicaría por la mayor masa de TAN disponible para ser oxidada y la menor velocidad del agua en las tuberías que facilita el asentamiento de bacterias.

Tabla 3: Valor máximo, promedio ( \pm error estándar) y mínimo para $\mathrm{TAN}, \mathrm{NH}_{3}-\mathrm{N}, \mathrm{NO}_{2}-\mathrm{N}, \mathrm{pH}$,

Temperatura, $\mathrm{O}_{2}$ disuelto, Sólidos Suspendidos no Sedimentales, Sólidos disueltos y Sólidos

Suspendidos Sedimentales para cada tiempo de retención hidráulica (TRH de 3 y 6 horas).

\begin{tabular}{|c|c|c|c|c|c|}
\hline & Variable & $\begin{array}{c}\text { Estanque } 1 \\
\text { THR } 6 \mathrm{~h}\end{array}$ & $\begin{array}{c}\text { Estanque } 4 \\
\text { THR 6h }\end{array}$ & $\begin{array}{c}\text { Estanque } 2 \\
\text { THR } 3 \mathrm{~h}\end{array}$ & $\begin{array}{c}\text { Estanque } 3 \\
\text { THR } 3 \mathrm{~h}\end{array}$ \\
\hline \multirow{9}{*}{ 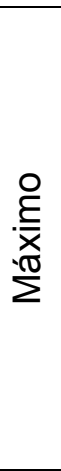 } & TAN (mg/l) & 3,14 & 3,24 & 1,71 & 1,86 \\
\hline & $\mathrm{NH}_{3}-\mathrm{N}(\mathrm{mg} / \mathrm{l})$ & 0,007 & 0,006 & 0,004 & 0,006 \\
\hline & $\mathrm{NO}_{2}-\mathrm{N}(\mathrm{mg} / \mathrm{l})$ & 0,102 & 0,058 & 0,037 & 0,016 \\
\hline & $\mathrm{pH}$ & 7,04 & 7,06 & 7,04 & 7,12 \\
\hline & Temp. $\left({ }^{\circ} \mathrm{C}\right)$ & 19,7 & 19,9 & 19,8 & 19,9 \\
\hline & $\mathrm{O}_{2}(\mathrm{mg} / \mathrm{l})$ & 7,11 & 7,31 & 6,44 & 7,13 \\
\hline & $\begin{array}{l}\text { S.S no Sedimentales } \\
(\mathrm{mg} / \mathrm{l})\end{array}$ & - & 16,43 & - & 16,23 \\
\hline & Sólidos disueltos $(\mathrm{mg} / \mathrm{l})$ & - & 13,93 & - & 12,43 \\
\hline & S.S Sedimentales (mg/l) & - & 51,38 & - & 52,00 \\
\hline \multirow{9}{*}{ 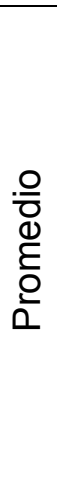 } & TAN (mg/l) & $2,35 \pm 0,408$ & $2,34 \pm 0,793$ & $1,18 \pm 0,256$ & $1,36 \pm 0,296$ \\
\hline & $\mathrm{NH}_{3}-\mathrm{N}(\mathrm{mg} / \mathrm{l})$ & $0,003 \pm 0,003$ & $0,003 \pm 0,002$ & $0,001 \pm 0,002$ & $0,002 \pm 0,002$ \\
\hline & $\mathrm{NO}_{2}-\mathrm{N}(\mathrm{mg} / \mathrm{l})$ & $0,026 \pm 0,030$ & $0,018 \pm 0,019$ & $0,008 \pm 0,011$ & $0,008 \pm 0,005$ \\
\hline & $\mathrm{pH}$ & $6,89 \pm 0,09$ & $6,91 \pm 0,11$ & $6,90 \pm 0,12$ & $6,94 \pm 0,13$ \\
\hline & Temp. $\left({ }^{\circ} \mathrm{C}\right)$ & $18,2 \pm 1,1$ & $18,3 \pm 1,1$ & $18,3 \pm 1,2$ & $18,3 \pm 1,2$ \\
\hline & $\mathrm{O}_{2}(\mathrm{mg} / \mathrm{l})$ & $5,67 \pm 0,2$ & $5,87 \pm 0,72$ & $5,32 \pm 0,58$ & $5,81 \pm 0,66$ \\
\hline & $\begin{array}{l}\text { S.S no Sedimentales } \\
(\mathrm{mg} / \mathrm{l})\end{array}$ & - & $11,93 \pm 2,81$ & - & $12,07 \pm 3,63$ \\
\hline & Sólidos disueltos $(\mathrm{mg} / \mathrm{l})$ & - & $9,46 \pm 3,03$ & - & $9,56 \pm 2,52$ \\
\hline & S.S Sedimentales (mg/l) & - & $40,80 \pm 10,02$ & - & $39,30 \pm 9,74$ \\
\hline \multirow{9}{*}{ : } & TAN $(\mathrm{mg} / \mathrm{l})$ & 1,67 & 1,16 & 0,89 & 0,96 \\
\hline & $\mathrm{NH}_{3}-\mathrm{N}(\mathrm{mg} / \mathrm{l})$ & 0,000 & 0,000 & 0,000 & 0,000 \\
\hline & $\mathrm{NO}_{2}-\mathrm{N}(\mathrm{mg} / \mathrm{l})$ & 0,008 & 0,000 & 0,000 & 0,000 \\
\hline & $\mathrm{pH}$ & 6,76 & 6,76 & 6,73 & 6,77 \\
\hline & Temp. $\left({ }^{\circ} \mathrm{C}\right)$ & 16,6 & 16,6 & 16,6 & 16,6 \\
\hline & $\mathrm{O}_{2}(\mathrm{mg} / \mathrm{l})$ & 4,80 & 5,11 & 4,57 & 5,01 \\
\hline & $\begin{array}{l}\text { S.S no Sedimentales } \\
(\mathrm{mg} / \mathrm{l})\end{array}$ & - & 7,57 & - & 7,12 \\
\hline & Sólidos disueltos $(\mathrm{mg} / \mathrm{l})$ & - & 4,30 & - & 4,60 \\
\hline & S.S Sedimentales (mg/l) & - & 30,58 & - & 27,50 \\
\hline
\end{tabular}

Los valores de TAN que presentaron las dos experiencias se encontraron por sobre lo recomendado para la especie. Sin embargo, creemos que esta situación no generó un efecto negativo en el crecimiento de los peces, ya que gracias al $\mathrm{pH}$ promedio obtenido inferior a 7, las concentraciones de $\mathrm{NH}_{3}-\mathrm{N}$ se mantuvieron en un promedio de 10 veces por debajo del nivel máximo recomendado de 0,02 mg/l (Muir, 1982; Lawson, 1995; Timmons et al., 2002). Además, producto del pH, los resultados indican que en promedio el $99,8 \%$ del valor de TAN obtenido corresponde a amonio ionizado $\left(\mathrm{NH}_{4}{ }^{+}\right.$ 
$\mathrm{N})$, que es considerado no tóxico para organismos acuáticos, excepto en concentraciones muy altas (Lawson, 1995). El nulo o escaso efecto del TAN, se refleja en los resultados de crecimiento y evolución positiva del factor de condición $(\mathrm{K})$, y específicamente en los porcentajes de crecimiento obtenidos en la experiencia de TRH de $6 \mathrm{~h}$ (sobre el 47\%), la que presentó los valores más altos de TAN. Un resultado similar obtuvo Summerfelt et al. (2004), quienes trabajaron con truchas en un sistema de re-uso parcial de agua, alcanzando concentraciones de TAN de 2,7 $\pm 0,07 \mathrm{mg} / \mathrm{l} \mathrm{sin}$ reportar problema en los peces.

Igualmente, las concentraciones de $\mathrm{NO}_{2}-\mathrm{N}$ se mantuvieron en un promedio de 10 veces por debajo del nivel máximo recomendado de 0,1 mg/l (Muir, 1982; Lawson, 1995) y en el caso de los SST, entre los rangos máximos recomendados por Lawson (1995) de $80 \mathrm{mg} / \mathrm{l}$ y los $40 \mathrm{mg} / \mathrm{l}$ sugeridos por Muir (1982). En relación al nivel de oxígeno disuelto, presente en los estanques, su valor promedio durante todas las experiencias $(5,66 \pm 0,54 \mathrm{mg} / \mathrm{l})$ se mantuvo cerca del límite inferior de lo recomendado para la especie, entre 6 a 8 mg/l (Lawson 1995; Timmons et al., 2002), y por sobre la concentración crítica de $2,5 \mathrm{mg} / \mathrm{l}$ para una temperatura de entre $19^{\circ} \mathrm{C}$ y $20^{\circ} \mathrm{C}$ (Wheaton, 1993).

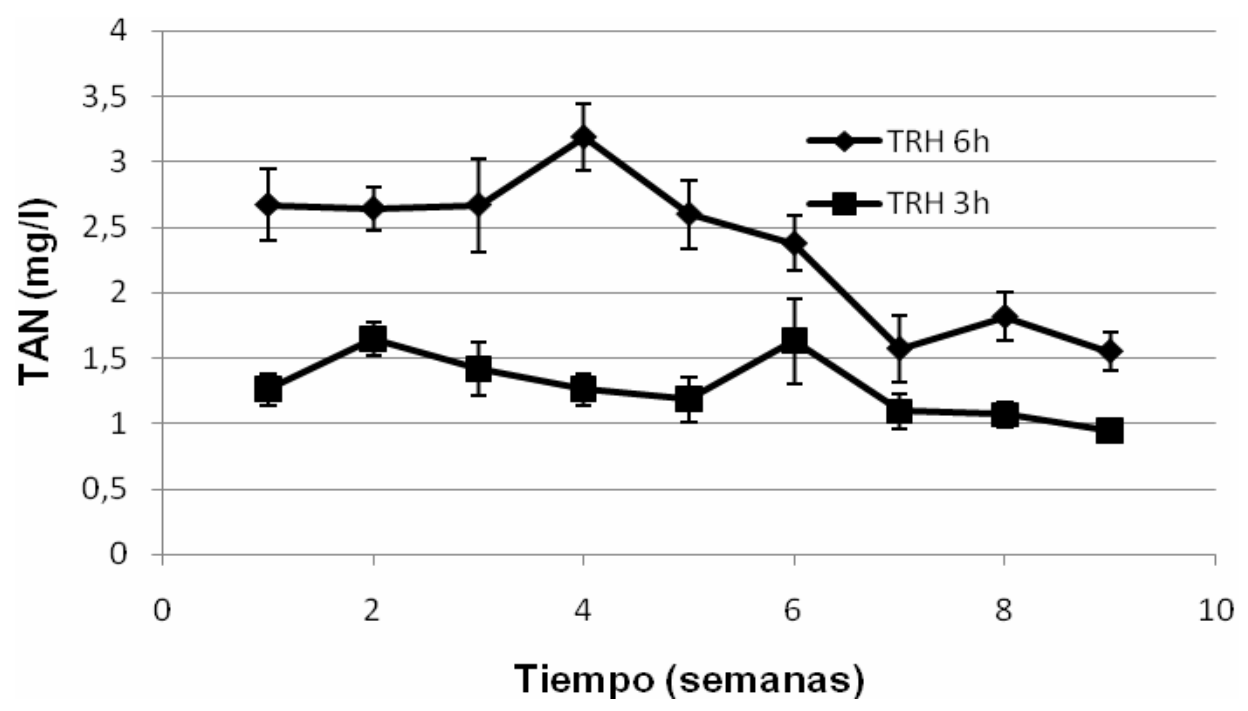

Fig. 3: Concentraciones de TAN (mg/l) observadas durante la experiencia para dos valores de TRH de agua. Se indica el error estándar $(n=22)$.

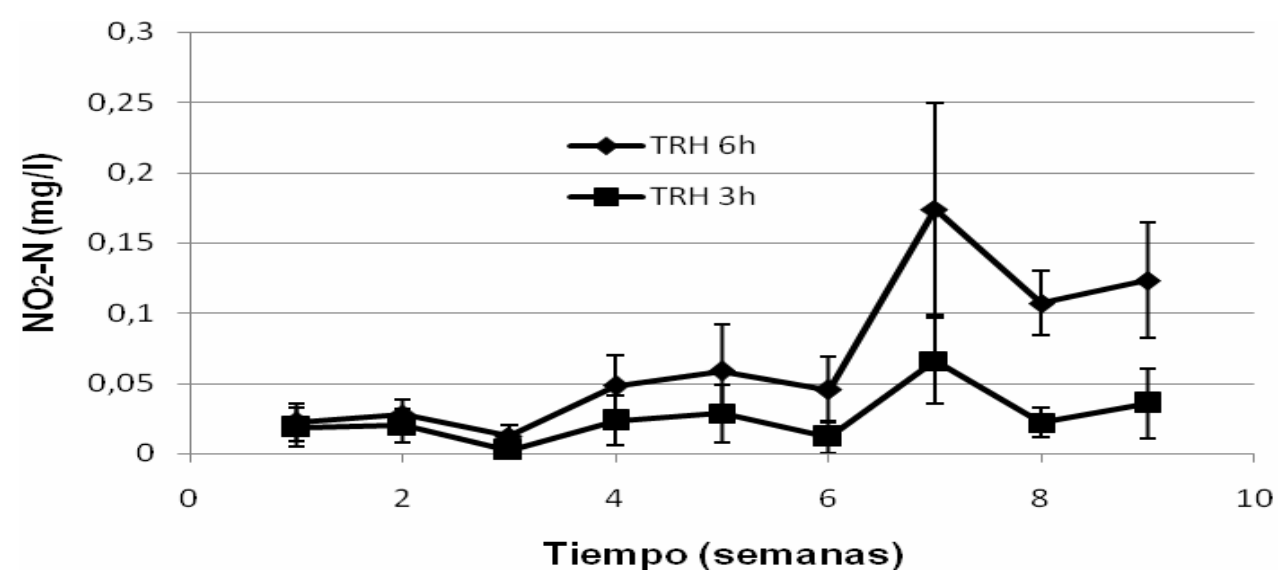

Fig. 4: Concentraciones de $\mathrm{NO}_{2}-\mathrm{N}$ (mg/l) observadas durante todas las semanas de la experiencia para dos valores de TRH de agua. Se indica el error estándar $(n=22)$

De esta forma, los resultados indican que los cambios en la concentración de las variables de calidad de agua analizadas, producto de los distintos $\mathrm{TRH}$, no sobrepasaron o se mantuvieron en los rangos de variación adecuados para el cultivo de la trucha arcoíris. 
Además, al contrastar los resultados promedios de calidad de agua obtenidos en este trabajo (Tabla 3 ), con rangos de variación normales y característicos de efluentes de pisciculturas de trucha, en modalidad de un paso, recopilados y reportados en Stewart et. al. (2006), los valores obtenidos se encuentran dentro del rango de variación normal reportado, en cuanto a $\mathrm{pH}(5,9$ a 9,4), oxígeno disuelto (5,4 a 14,3 mg/l), SST (1 a $97 \mathrm{mg} / \mathrm{l}), \mathrm{NO}_{2}-\mathrm{N}$ (inferior a 0,02 mg/l) y TAN $(0,02$ a 1,53 mg/l), salvo esta última variable en la experiencia de TRH de $6 \mathrm{~h}$.

\section{Efecto del tiempo de retención hidráulico (TRH) en la productividad del sistema}

Al comparar los resultados obtenidos en los distintos TRH con un sistema tradicional de cultivo de truchas de un paso (información adaptada de Colt, 1991), los beneficios por ahorro en consumo de agua son evidentes (Tabla 4). La comparación indica que para iguales densidades de cultivo (entre 15 a $40 \mathrm{~kg} / \mathrm{m}^{3}$ ), la Carga de Cultivo (C) es 2,5 veces mayor para un TRH de 3 y de 5 veces para un TRH de $6 \mathrm{H}$, frente a los sistemas de un paso que consideran sólo la aireación y el uso de oxígeno puro para aumentar el tiempo de vida útil del agua. Esto implica que es factible producir entre 2,5 a 5 veces más kilos de peces por litro de agua que en un sistema tradicional, a igual densidad de cultivo, cuando se trabaja con un TRH de 3 h ó $6 \mathrm{~h}$. Esto es relevante para centros de cultivo que engordan en tierra trucha arcoíris y cuya producción se encuentra limitada por la disponibilidad de agua fresca, ya que les permitiría usar el remanente de agua que genera un TRH mayor, para aumentar los volúmenes de producción, manteniendo condiciones de calidad de agua adecuadas para la especie. Además, el uso en los sistemas de un paso con un TRH mayor al normalmente utilizado $(0,17 \mathrm{~h}$ a $0,96 \mathrm{~h}$ ), puede facilitar la utilización de fuentes de agua que por su bajo caudal disponible (pozos o riachuelos), no permiten actualmente el desarrollo de una actividad acuícola económicamente rentable.

Tabla 4: Comparación de la productividad en el uso del agua de diferentes Sistemas de Cultivo de Un Paso y TRH.

\begin{tabular}{|c|c|c|c|c|c|}
\hline $\begin{array}{c}\text { Sistemas } \\
\text { de Cultivo } \\
\text { de Un Paso }\end{array}$ & $\mathrm{TRH}(\mathrm{h})$ & $\begin{array}{c}\text { Densidad } \\
\left(\mathrm{kg} / \mathrm{m}^{3}\right)\end{array}$ & $\begin{array}{c}\text { Carga } \\
\text { kg de pez/litro } \\
\text { por minuto }\end{array}$ & Especie & Autor \\
\hline $\begin{array}{c}\text { Sin } \\
\text { Aireación }\end{array}$ & $\begin{array}{c}0,168- \\
0,336\end{array}$ & $15-30$ & $0,5-2$ & $\begin{array}{l}\text { Salmón y } \\
\text { Trucha }\end{array}$ & Colt et al.,1991 \\
\hline $\begin{array}{c}\text { Con } \\
\text { aireación }\end{array}$ & $\begin{array}{c}0,336- \\
0,48\end{array}$ & $20-40$ & $1-3$ & $\begin{array}{l}\text { Salmón y } \\
\text { Trucha }\end{array}$ & Colt et al.,1991 \\
\hline $\begin{array}{c}\text { Con } \\
\text { aireación }\end{array}$ & 3 & $30-40$ & 7,8 & Trucha & $\begin{array}{l}\text { Experiencia } \\
\text { actual }\end{array}$ \\
\hline $\begin{array}{c}\text { Con } \\
\text { aireación }\end{array}$ & 6 & $30-40$ & 15,6 & Trucha & $\begin{array}{l}\text { Experiencia } \\
\text { actual }\end{array}$ \\
\hline $\begin{array}{l}\text { Oxigeno } \\
\text { puro }\end{array}$ & $0,48-0,96$ & $30-50$ & $2-6$ & $\begin{array}{l}\text { Salmón y } \\
\text { Trucha }\end{array}$ & Colt et al.,1991 \\
\hline $\begin{array}{c}\text { Sin } \\
\text { Aireación }\end{array}$ & NR & NR & $0,8-1,4$ & $\begin{array}{c}\text { Peces agua } \\
\text { fría }\end{array}$ & Pillay (1982) \\
\hline $\begin{array}{c}\text { Sin } \\
\text { Aireación }\end{array}$ & NR & NR & 1,4 & $\begin{array}{c}\text { Peces agua } \\
\text { fría }\end{array}$ & $\begin{array}{l}\text { Piedrahita } \\
\text { (2003) }\end{array}$ \\
\hline
\end{tabular}

\section{CONCLUSIONES}

Bajo las condiciones de este estudio, no existe diferencia significativa en el crecimiento de trucha arcoíris (O.mykiss), durante su engorda, si su cultivo se realiza en un sistema de un paso con un TRH de 3 h ó $6 h$.

El efecto de dilución que ambos TRH generan sobre la acumulación de desechos metabólicos tóxicos en el agua (amoniaco y nitrito), permite mantener las concentraciones promedio de estos compuestos en un valor de 10 veces menor a lo recomendado, tanto para $3 \mathrm{~h}$ y $6 \mathrm{~h}$ 
Si bien la temperatura del agua y las concentraciones de TAN, que gracias al valor de $\mathrm{pH}$ se constituía en promedio en un $99,8 \%$ de amonio ionizado, se encontraron por sobre los recomendado para la especie, los resultados de crecimiento (entre un $49 \%$ y $34 \%$ en 60 días), las bajas concentraciones de desechos metabólicos tóxicos y el aumento del factor de condición (K) de los individuos en cultivo (22\% promedio para las dos experiencias), indican que su efecto no fue significativo.

Sobre esta base el uso de sistemas de cultivo de un paso para la engorda de trucha arcoíris con TRH de 3 h ó 6 h, permitirían aumentar la eficiencia del uso del recurso agua y la productividad del sistema entre 2,5 a 5 veces, en términos de biomasa producida por litro de agua.

La implementación de estos resultados permitiría mejorar la productividad de los centros de cultivo dedicados a la engorda de truchas, reducir los consumos de agua que son críticos en esta etapa de producción y utilizar fuentes de agua que por su bajo caudal disponible (pozos o riachuelos), no permiten actualmente el desarrollo de una acuícola económicamente rentable.

\section{REFERENCIAS}

APHA, American Public Health Association. In: Clesceri, Greenberg, Trussell (Eds.), Standard Methods for the Examination of Water and Wastewater, 20th ed. Washington, DC. (1998)

Barnabé, G; Acuicultura Volumen I. 1-478 p. Ed. Ediciones Omega, S.A. (1991)

Cho, C.Y; Feeding systems for rainbow trout and other salmonids with reference to current estimates of energy and protein requirements, Aquaculture: 100, 107-123 (1992).

Colt, J.E.; Aquaculture production systems. Journal of Animal, Science: 69, 4183-4192 (1991).

Colt, J.E., K. Orwicz y G. Bouck; Water quality considerations and criteria for hight-density fish culture with supplemental oxygen. In Colt, J., White, R.J. (Eds.), Fisheries Bioengineering Symposium 10. American Fisheries Society, Bethesda, Maryland, pp. 372-385 (1991).

Jobling, M., O.H. Meløy, J. dos Santos y B. Christiansen; The compensatory growth response of the Atlantic cod effects of nutritional history. Aquaculture International: 2, 75-90 (1994).

Jobling, M; The thermal growth coefficient (TGC) model of fish growth: a cautionary note. Aquaculture Research: 34, 581-584 (2003).

Lawson, T.B; Fundaments of Aquacultural Engineering, $1^{\text {a }}$ edición, International Thomson Editores, New York, USA (1995).

Lyssenko, C. y F. Wheaton; Impact of rapid impulse operating disturbances on ammonia removal by trickling and submerged-upflow biofilters for intensive recirculating aquaculture. Aquacultural Engineering: 35, 38-50 (2006).

Meade, J.W; Intensity of aquaculture production, definitions, meanings and measures. Engineering Aspecs of Intensive Aquaculture, Proceeding from the Aquaculture Conference, Ithaca, NY: Cornell University, April 4-6 (1991).

Muir, J.F; Recirculated system in aquaculture, In: Muir, J.F., Roberts, R.J. (Eds.). Recent Advances I Aquaculture, Vol 1, 453 pp, Croom Helm and Westview Press. London, England (1982).

Piedrahita, R; Reducing the potential environmental impact of tank aquaculture effluents through intensification and recirculation. Aquaculture: 226, 35-44 (2003).

Pillay, T.V.R; Aquaculture and the Environment, 189 pp, Fishing News Books (Ed), Oxford, England, (1982). 
Piper, R.E. y otros 6 autores; Hatchery Management, 218 pp., U.S. Fish and Wildlife service, Washington, DC. (1982).

Stewart, N.T, G.D. Boardman y L.A. Helfrich; Treatment of rainbow trout (Oncorhynchus mykiss) raceway effluent using baffled sedimentation and artificial substrates. Aquacultural Engineering: 35 , 166-178 (2006)

Summerfelt, S.T. y otros 4 autores; A partial-reuse system for coldwater aquaculture, Aquacultural Engineering: 31, 157-181 (2004).

Timmons, M.B. y otros 4 autores; Sistema de Recirculación para Acuicultura. pp. 19-39 (2002).

Tucker, C.S. y E.H. Robinson; Channel Catfish Farming HandBook. New York, Van Nostrand Reinhold (1990).

Wheaton, F; Acuacultura. Diseño y Construcción de Sistemas, $1^{\text {a }}$ edición, 703 pp, A.G.T. Editor S.A., Planta Alta, México (1993). 